\title{
Érotisme, désir et sadisme chez Sartre
}

JEAN-PIERRE BOULE

Résumé: Cet article se concentre sur l'érotisme et sur les relations sexuelles dans les écrits et la biographie de Sartre, et en particulier sur la notion de sadisme, explorant l'hypothèse que la biographie de Sartre sur Charles Baudelaire ainsi que Faut-il brûler Sade? de Simone de Beauvoir nous aident à explorer indirectement érotisme, désir et sadisme chez Sartre. Le texte est appuyé par une variété de sources secondaires, en particulier par des articles de Christina Howells et de Serge Doubrovsky. L'accent est mis sur la matérialité physique de l'acte sexuel et de l'érotisme mais sans jamais négliger les structures psychologiques et existentielles du sadisme et du masochisme.

Mots-clés: Charles Baudelaire, érotisme, Jean-Paul Sartre, Marquis de Sade, sadisme, Simone de Beauvoir, théorie des relations d'objet

Dans un entretien avec Michel Contat publié en 1975, Sartre admet ne pas avoir parlé ouvertement de sa sexualité dans son œuvre, indiquant qu'il ne voit aucune raison de s’exécuter sauf dans une autre société où tout le monde le ferait. Contat rétorque que ses romans contiennent de nombreux indices sur sa sexualité. Sartre acquiesce et renchérit: il faut aussi inclure ses ouvrages philosophiques. Mais d'ajouter que tous ces livres ne représentent qu'un moment de sa vie sexuelle qui n'y est pas décrite avec suffisamment de détails et de complexité pour qu'on puisse l'y trouver vraiment. ${ }^{1}$ Si Sartre n'a pas parlé ouvertement de sa sexualité, il en a pourtant laissé des traces. Les relations avec autrui sont étudiées dans L'Être et le Néant y compris les rapports sexuels. Dans la fiction, on trouve de nombreux passages sur la sexualité. 
Nous avons également de multiples interviews qui portent sur le sujet, le film Sartre par luimême, ainsi que les entretiens avec Simone de Beauvoir d'août-septembre 1974 où les deux philosophes reviennent longuement sur la question. Enfin, il existe des articles de critiques, notamment un article de Christina Howells qui étudie le phénomène du désir à partir des concepts d'en-soi et de pour-soi, sur le terrain de l'ontologie phénoménologique: 'Desire desires the other as in-itself-for-itself. The other, similarly, desires to be in-itself-for-itself. This is the original useless passion'. ${ }^{2}$ On compte également un article retentissant écrit par Serge Doubrovsky qui dans 'Sartre: Retouches à un autoportrait (une autobiographie visqueuse)'3 démontre que la sexualité de Sartre est cachée dans les pages de L'Être et le Néant, notamment avec sa description de l'Obscène dans la section intitulée 'L'existence d'autrui'.

En utilisant un livre de Sartre sur Baudelaire publié en 1963 (Baudelaire) et surtout un livre de Beauvoir sur le marquis de Sade publié en 1955 (Faut-il brûler Sade?), le présent article souhaite apporter un éclairage nouveau sur l'érotisme de Sartre en resserrant ce débat à partir du postulat suivant: en parlant de Baudelaire, Sartre projetterait (en partie) sa propre sexualitét; en parlant de la sexualité de Sade, Beauvoir s'efforcerait de comprendre (et parlerait en partie de) la sexualité de Sartre. ${ }^{5}$ Dans un premier temps, nous allons situer Sade dans le contexte des années 1950 puis nous pencher sur les raisons qui ont motivé Beauvoir à écrire sur cet auteur avant de tenter de définir la relation d'objet, y compris chez Beauvoir et chez Sartre. Dans un deuxième temps, nous interrogerons Baudelaire et Sade non sans avoir tout d'abord esquissé le concept de sadisme. À partir de là, 'érotisme, désir et sadisme chez Sartre' commenceront à se profiler à grands traits. La majeure partie de l'article s'efforcera d'affiner ce portrait. Pour ce faire, biographie, entretiens, littérature, philosophie, carnets, correspondance, cahiers de jeunesse ... seront convoqués et mis en dialogue avec Baudelaire et Faut-il brûler Sade? 


\section{Pourquoi Sade?}

Dans les années 1950, Sade était un objet d'études en France. Les surréalistes avaient commencé à le réhabiliter dans les années 1920. Les éditions Pauvert avaient entamé dès 1947 la publication de ses œuvres complètes. Pierre Klossowski publiait la même année Sade mon prochain où l'athéisme sadien lui paraissait foncièrement inspiré par son opposition radicale à la théologie. ${ }^{6}$ En 1949, Lautréamont et Sade de Maurice Blanchot soulignait l'exigence d'une dialectique au sens moderne chez le marquis de Sade, pour affirmer l'homme intégral 'l'homme unique dans son genre'. ${ }^{7}$ Georges Bataille, qui écrivit que si Sade n'avait pas existé, il aurait fallu l'inventer, fit plus que quiconque pour asseoir sa réputation dans l'histoire des idées. ${ }^{8}$ Pourquoi Beauvoir décida-t-elle de se consacrer à Sade? Plusieurs réponses à cette interrogation. Elle le décrit comme un 'grand moraliste' (55). Elle le voit également en précurseur de la lutte des classes ainsi que 1'analyse Ursula Tidd: 'In the theatre of the erotic, Beauvoir contends that Sade demonstrated how the interests of master and slave can be irreconcilable and, in so doing, he foresaw the class struggle'. ${ }^{9}$ Judith Butler pense qu'en étudiant un auteur qui refuse de reconnaître la présence d'Autrui dans l'échange sexuel, Beauvoir a l'occasion d'affirmer les structures de l'intersubjectivité telles qu'elles se manifestent dans cet échange. ${ }^{10}$ Bergoffen situe ce choix au niveau de la morale beauvoirienne dans la mesure où c'est avec son corps (l'endroit le plus vulnérable) que Sade s'oppose à la société. Il incarne 'the point of the excessive, erotic body ${ }^{11}$ même si Beauvoir sera contrariée par le fait que Sade pervertit ce corps érotique. ${ }^{12}$ Comme nous allons le découvrir dans cet article, il y a de telles similarités entre l'approche de l'érotisme chez Sade et chez Sartre que, même inconsciemment, en écrivant sur Sade, Beauvoir comprend mieux Sartre, de même qu'en étudiant Baudelaire, Sartre comprend 
mieux son propre érotisme, (s')avouant peut-être certaines de ses pratiques ou préférences sous couvert du 'poète maudit', ce qui serait un moyen de les 'normaliser'.

\section{La relation d'objet}

Afin de situer nos remarques à venir, quelques réflexions théoriques sur la relation d'objet sont de rigueur dans la mesure où toute théorie sur l'amour est par nécessité une théorie sur les relations d'objet: comment un sujet peut-il aimer un autre sujet qui, par le fait d'être aimé (passivité), devient un objet de l'amour de l'autre? Dans L'Être et le Néant, Sartre déclare dès le début du chapitre 'Les relations concrètes avec autrui' que le conflit est le sens originel de l'êtrepour-autrui. ${ }^{13}$ Selon Leo Bersani et Adam Phillips dans un ouvrage récent, Intimacies: 'Love is transitive; to conceptualize it is to address $[\ldots]$ the very possibility of a subject loving an object'. ${ }^{14}$ Comme le dit Bersani, depuis Trois essais sur la théorie sexuelle de Freud, la psychanalyse a toujours été sceptique quant à cette possibilité. ${ }^{15}$ Dans ledit ouvrage, Freud exposait que l'on ne peut pas séparer l'amour de la faculté de mémoire, pointant l'image du nouveau-né s'allaitant au sein maternel comme prototype de l'amour. Lorsque l'on trouve l'objet aimé, en fait on le retrouve sous la forme du sujet aimé. Cet argument introduit le narcissisme du sujet dans la relation d'objet. ${ }^{16}$ Cette structure fondamentalement narcissique est réaffirmée par Jacques Lacan dans Les Quatre concepts fondamentaux de la psychanalyse.${ }^{17}$ De façon à réconcilier les deux positions antithétiques, Bersani propose le concept de 'narcissisme impersonnel' ('impersonal narcissism') où se fonde le nouveau pouvoir de l'amour. Cette nouvelle forme de narcissisme implique de ne plus utiliser l'opposition entre le même et l'autre exprimée comme différence en tant que structure catégorisant les êtres humains. ${ }^{18}$ 
Quelle est la position de Beauvoir sur la relation d'objet telle qu'elle ressort de Faut-il brûler Sade? Par le trouble, l'existence est saisie en soi et chez l'autre comme subjectivité et passivité à la fois. Il en résulte qu' 'à travers cette unité ambiguë les deux partenaires se confondent; chacun est délivré de sa présence à soi et atteint une communication immédiate avec l'autre' (33); deux sujets arrivent à coexister dans la jouissance. Partant de la subjectivité et de la passivité, la jouissance génère deux sujets qui arrivent à communiquer. Qu'en est-il de Baudelaire et de Sade? Selon Sartre, Baudelaire fait face à une même difficulté de narcissisme que celle soulignée par Bersani. Dans le plaisir normal, on jouit de l'objet (notons en passant que Sartre utilise 'objet' et non 'sujet') et on s'oublie; dans l'attitude de Baudelaire qu'il qualifie de 'titillation énervante', c'est du désir qu'on jouit, c'est-à-dire de soi (239). Beauvoir lance son verdict sur Sade dès le début du livre: 'C'est au contraire l'alliance d'appétits sexuels ardents avec un «isolisme » affectif radical qui m'apparait comme la clé de son érotisme' (32). Cet ‘isolisme' affectif sera plus loin qualifié d'autisme avec, il est vrai, une définition peu orthodoxe. ${ }^{19}$

\section{La non-réciprocité}

Il s'agit de définir le concept de sadisme tel qu'il est utilisé par Beauvoir dans un livre écrit dans les années 1950. Beauvoir elle-même nous invite à la prudence à cause de la diversité des significations offerte par ce concept. Sachant très bien que sadisme signifie cruauté ('fustigations, saignées, tortures, meurtres', 30), Beauvoir se positionne au niveau de la conscience. Le sadisme qui l'intéresse et qui caractérise Sade, c'est la tension d'une volonté qui s'applique à réaliser la chair sans se perdre en elle (39). Notre travail adoptera cette définition 
tout en reconnaissant que nous sommes loin du genre de définition hétérogène pratiquée de nos jours par exemple par Gayle Rubin. ${ }^{20}$

Selon Beauvoir, il y a une absence chez le 'sadique' qu'il s'efforce de compenser par le sadisme: il ne peut jamais s'oublier et n'arrive pas à réaliser la présence d'autrui. Il y a une séparation radicale entre l'autre-objet et le sujet-souverain. La cruauté du sadique lui 'permet de saisir à travers l'autre l'unité conscience-chair et de la projeter en soi' (41). Le sadique se sert de l'autre pour ressentir indirectement ce qu'il ne peut ressentir en tant qu'expérience vécue, ainsi 'la confusion du pour-soi et du pour-autrui s'accomplit' (43-44). Ce que Beauvoir appelle sadisme pourrait être perçu comme une simple perversion, dans le sens étymologique de corruption, d'altération d'un contrat de réciprocité qui est rompu. Et cette forme de perversion, l'homme Sartre la revendique, notamment dans ses entretiens avec Beauvoir :

mais la réciprocité était la chose que je sentais le moins; le fait que l'autre personne pouvait également avoir du plaisir à sentir mon corps. Par exemple, quand j'étais dans les bras d'une personne [...] je sentais moi saisissant la chair librement, mais non pas l'autre personne saisissant mon corps. ${ }^{21}$

L'écrivain l'évoque également. On lit par exemple dans L'Âge de raison la description suivante dans une scène entre Mathieu et Marcelle:

Il l'attira contre lui: il n'avait pas exactement de désir pour elle en cet instant, c'était plutôt l'envie de voir cet esprit rétif et anguleux fondre comme une aiguille de glace au soleil [...] il se pencha sur elle avec une sorte de malaise: il aurait voulu s'oublier 
et l'oublier. Mais il y avait beau temps qu'il ne s'oubliait plus quand il faisait

l'amour avec elle. ${ }^{22}$

Christina Howells repère plusieurs exemples d'échec du désir dans sa production romanesque. ${ }^{23}$

Le philosophe revendique également cette forme de perversion. Dans L'Être et le Néant, Sartre expose qu'en voulant m'approprier la liberté d'autrui, cette liberté s'effondre sous mon regard de sorte que je suis condamné à rechercher la liberté d'autrui à travers l'objet qu'il est pour moi. Je trouve alors 'des conduites privilégiées qui pourraient m'approprier cette liberté à travers une appropriation totale du corps d'autrui' (430); Sartre ajoute que ces tentatives sont vouées à l'échec. Un peu plus loin, il affirmera que ma tentative originelle pour me saisir de la subjectivité libre de l'autre est 'le désir sexuel' (432), désir qui passe par la caresse: 'Le désir s'exprime par la caresse comme la pensée par le langage' (432). Il liera ensuite ce désir sexuel à la facticité et à un consentement passif au désir (438). Alors qu'il travaillait à la fin des années 1940 à un ouvrage qui devait s'intituler L'Homme (qui ne vit jamais le jour), Sartre écrivit un texte sur l'amour courtois qui sera publié en 2007. Même dans ce concept on retrouve l'anéantissement de la conscience sans qu'il y ait nécessairement de rapport sexuel, au niveau de l'âme: 'Ainsi l'entrée en amour est une mort. En donnant ou en se laissant ravir le moi empirique, comme dans tous les mystères, on meurt'. ${ }^{24}$

L'attitude de Sartre est corroborée par Beauvoir dans ses Cahiers de jeunesse. Elle note que, dans leurs étreintes, Sartre ne se donne pas aussi pleinement qu'elle. ${ }^{25}$ Elle confiera à John Gerassi que Sartre avait de grandes difficultés à atteindre l'orgasme mais que ses érections duraient très longtemps. Faire l'amour avec lui était épuisant, précisément parce qu'il ne voulait 
pas s'abandonner. ${ }^{26}$ Dans son livre, Mémoires d'une jeune fille dérangée, Bianca Lamblin (Bianca Bienenfeld, appelée dans la fiction 'Louise Védrine’) raconte comment Sartre prit sa virginité en 1939. Elle le décrit ainsi pendant leur premier rapport sexuel: 'Son intelligence toujours vigilante brisait tous les liens entre son esprit et son corps. Sans doute les choses liées au corps lui semblaient-elles étrangères'. ${ }^{27}$ Chez le héros sadique, Beauvoir arguë qu'il n'existe pas de métamorphose du corps en chair car 'pas un instant il ne se perd dans son animalité: il demeure si lucide, si cérébral' (32). Dans une lettre à Beauvoir écrite en février 1940, Sartre avoue qu'il n'a pas de sensualité. ${ }^{28}$ Mais ne nous laissons pas emporter par ce raisonnement... Doubrovsky dit bien que des conditions sexuelles 'sadiques' ne dénotent pas une absence, plutôt une forme particulière, 'les psychiatres diraient "perverse" de sensualité'. ${ }^{29}$

\section{Les rapports sexuels}

En 1974, les entretiens avec Beauvoir reviennent longuement sur la question des rapports sexuels. Sartre confirme que l'objectivité et le fait d'être actif prenaient toujours le pas sur la subjectivité et la passivité (401). Il explique que les rapports sexuels étant obligés ('le sexuel c'est “coucher avec"' 385), il s'exécutait mais n'y attachait pas une telle importance ('Juste un petit plaisir à la fin mais assez médiocre' 400). On remarque une autre similarité avec Baudelaire qui, selon la projection de Sartre, privilégie l'irritation nerveuse du désir à son assouvissement (159), concluant: 'sans doute la possession physique [...] ne l'attirait pas particulièrement' (95). Nous avons vu que Beauvoir introduisait la notion de sadisme dans sa description de Sade. Elle évoquera un 'corps froid, tendu, rebelle à tout envoûtement' (32). Toujours dans les entretiens, la philosophe parle des côtés actifs et passifs qui doivent être développés chez les deux amants, soulignant que chez Sartre seul le côté actif comptait, avant de constater : 'ce qui vous amenait à 
la maîtrise de vous-même, mais en même temps à une certaine froideur' (415). Ce dernier acquiesce avant d'ajouter que l'on pourrait parler chez lui d'un tout petit peu de sadisme puisque la personne était donnée et que lui ne l'était pas. Beauvoir lui fait préciser sa pensée et Sartre s'exécute. Ce qu'il qualifie de sadique, c'est bien le fait que l'un soit pure activité et l'autre pure passivité. L'Autre est réduit à l'état d'objet alors que l'on s'attendrait à une vraie réciprocité (415).

Et que dit Sartre à Beauvoir, sans ambages?

Alors, comme j'étais convenablement sexué, je bandais rapidement, facilement; je faisais l'amour souvent, mais sans un très grand plaisir. Juste un petit plaisir à la fin, mais assez médiocre. J'aimais mieux être en liaison avec le corps tout entier, caresser le corps, bref être actif [...] que faire l'amour proprement dit (400).

Sartre interprète l'érotisme de Baudelaire comme suit: 'faire l'amour comme tout le monde: quelle insanité !' (138). Le poète maudit n'aime pas l'acte sexuel qui lui fait horreur parce qu'il est naturel et brutal et 'parce qu'il est, au fond, une communication avec l'Autre' (95). Et on lit dans L'Être et le Néant: 'Aussi, l'homme moyen ne peut-il, par paresse d'esprit et conformisme, concevoir d'autre fin à son désir que l'éjaculation' (435). Beauvoir renchérit sur Sade: 'Il n'a que mépris pour les pauvres efféminés qui possèdent normalement leurs épouses' (36). Beauvoir explique que si Sade a connu les sollicitations du désir, il semble ignorer l'expérience du trouble et jamais la volupté n'apparaît dans ses récits comme oubli de soi, pâmoison, abandon. La philosophe se base sur une lettre de Sade 'Vanille et manille' pour confirmer que Sade éprouvait l'orgasme comme une crise épileptique (31-32). Déjà, dans un article précédant 
Intimacies, Bersani écrivait: 'It is possible to think of the sexual as, precisely, moving between a hyperbolic sense of self and a loss of consciousness of self'. ${ }^{30}$ Dans L'Être et le Néant, le philosophe décrit le dernier degré du désir comme évanouissement en tant que 'dernier degré de consentement au corps' (439). Pour Sartre, Baudelaire - haïssant l'abandon de soi - pousse cette 'phobie' à son paroxysme en devenant impuissant par peur de trop jouir (238-239). Selon Beauvoir, Sade répugne à toute égalité que créerait une commune volupté car si la personneobjet qui lui sert de jouissance jouit à son tour, sa propre jouissance en sera dérangée, concluant: 'Toute jouissance partagée s'affaiblit' (33-34).

Si le philosophe a horreur du trouble pour lui-même, quid du sexe féminin? Le sexe féminin est qualifié d'obscène dans le traité philosophique et comparé à toute chose béante: 'C'est un appel d'être, comme d'ailleurs tous les trous [...] Sans aucun doute le sexe est bouche, et bouche vorace qui avale le pénis - ce qui peut bien amener l'idée de castration: l'acte amoureux est castration de l'homme - mais c'est avant tout que le sexe est trou' (676). ${ }^{31}$ Beauvoir fait remarquer que les personnages de Sade éprouvent un dégoût profond pour le 'devant' des femmes (35).

\section{Le sadisme}

Nous avons vu que Sartre dénonçait chez lui 'un tout petit peu de sadisme'. Qu'entend-il par là? Doubrovsky démontre que c'est en cherchant dans L'Être et le Néant que l'on en apprend plus ${ }^{32}$ :

Le sadique a ressaisi son corps comme totalité synthétique et centre d'action [...] il s'éprouve en face de l'autre comme pure transcendance; il a en horreur pour lui le trouble 
[...] Il veut la non-réciprocité des rapports sexuels, il jouit d'être puissance appropriante et libre en face d'une liberté captivée par la chair. (449-450)

Le problème réside dans le fait que Sade (ainsi que Sartre comme on l'a vu) éprouve des pulsions sexuelles envers ces objets étrangers auxquels il est incapable de s'unir et donc il lui faut inventer des manières singulières de les saisir (33).

Beauvoir explique l'attitude de Sade qui, comme on va le voir, fait écho à celle de Sartre et mérite donc d'être citée dans sa totalité:

Normalement, c'est par le vertige de l'autre fait chair que chacun s'envoûte dans sa propre chair. Si le sujet reste enfermé dans la solitude de sa conscience, alors il échappe à ce trouble et il ne peut rejoindre l'autre que par des représentations; un amant cérébral et froid épie avidement la jouissance de sa maitresse, et il a besoin de s'en affirmer l'auteur parce qu'il n'a pas d'autre moyen d'atteindre sa propre condition charnelle: on peut qualifier de sadique cette conduite qui compense la séparation par une tyrannie réfléchie.

Beauvoir dégage une idée essentielle de son analyse de Sade: le souci de s'affirmer l'auteur de la jouissance de l'autre. On lit dans L'Être et le Néant: 'La caresse fait naître Autrui comme chair pour moi et pour lui-même' (440). Dans ses entretiens avec le Castor, Sartre explicite: 'Autrement dit pour moi, le rapport essentiel et affectif impliquait que j'embrasse, que je caresse, que je promène mes lèvres sur un corps' (385). Avec une préférence pour la caresse: ‘Autrement dit, j'étais plutôt un masturbateur de femmes qu'un coïteur' (385). Sartre explique à son 
interlocutrice qu'à travers la caresse, c'était le côté actif qui l'intéressait, la position de la main et la sensation de la chair dans la mesure où il la faisait naître (400) et qu'il aimait mieux être en liaison avec le corps tout entier, caresser le corps, que de faire l'amour ajoutant: 'bref être actif avec les mains, avec les jambes, toucher la personne' (400). Dans L'Être et le Néant, Sartre approfondit la symbolique de la caresse: 'La caresse ne se veut pas simple contact [...] elle est façonnement. En caressant autrui, je fais naître sa chair par ma caresse, sous mes doigts' (440). Pour continuer à en savoir plus sur le 'sadisme' de Sartre qui, rappelons-le, a bien dit ne pas avoir parlé ouvertement de sa sexualité dans son œuvre, il faut se tourner de nouveau vers Doubrovsky. Ce dernier cite le passage précédant en démontrant que le texte théorique reproduit celui de l'aveu épistolaire puis le dépasse ${ }^{33}$ avant d'éclairer les choses en se tournant de nouveau vers le traité philosophique: 'Quant au type d'incarnation que le sadisme voudrait réaliser, c'est précisément ce que l'on nomme l'Obscène [...] L'obscène apparait lorsque le corps adopte des postures qui le déshabillent entièrement de ses actes et qui révèlent l'inertie de sa chair' (452453).

Il explicite ensuite:

Le sadique manie le corps de l'Autre, pèse sur ses épaules pour l'incliner vers la terre et faire ressortir ses reins, etc. L'idéal du sadique sera donc d'atteindre le moment où l'Autre sera déjà chair sans cesser d'être instrument ... où les cuisses, par exemple, s'offrent déjà dans une passivité obscène et épanouie et sont encore des instruments qu'on manie, qu'on écarte et que l'on courbe, pour faire saillir davantage les fesses et pour les incarner à leur tour. $(E \& N, 453)$ 
De façon à illustrer le texte philosophique, penchons-nous sur Erostrate, l'une des nouvelles écrites par Sartre et publiée en 1939. Le personnage principal Paul Hilbert invite une prostituée dans sa chambre. Il lui demande de se déshabiller alors qu'il reste vêtu puis la fait marcher, se promener dans la chambre. ${ }^{34}$ Ce qui l'excite correspond à la description de l'obscène dans la citation ci-dessus ( $E \& N, 452-453)$ ainsi qu'à une description antérieure: 'La vue d'un corps nu, de dos, n'est pas obscène. Mais certains dandinements involontaires de la croupe sont obscènes' $(E \& N, 452)$. Elle marche d'un air gauche car, nous dit le narrateur, rien n'embête plus les femmes que de marcher nues, surtout en posant les talons à plat: 'La putain voûtait le dos et laissait pendre ses bras. Pour moi, j'étais aux anges' ${ }^{35}$ Cette même nuit, il repensera à elle et à 'son ventre gras qui sautait à chacun de ses pas' ${ }^{36}$ Dans le film Sartre par lui-même, Bost raconte une anecdote venue de Sartre alors qu'il enseignait au Havre et avait été la veille au bordel. Le professeur de philosophie répétait le lendemain: 'Je suis monté sur le dos d'une robuste putain!', une autre allusion à l'obscène qui fait écho à Hilbert dans Erostrate: 'On leur monte dessus, c'est entendu, mais elles vous dévorent le bas-ventre avec leur grande bouche poilue' ${ }^{37}$

Afin d'établir un parallèle avec la scène dans Erostrate, tournons-nous vers le Baudelaire. Selon Sartre, il préfère les plaisirs à distance à la pénétration: 'Voir, palper, respirer la chair de la femme' (95). Le philosophe est sûr de lui: il affirme que Baudelaire était voyeur et fétichiste (96). Voyeur, car il peut être parcouru par un frisson obscène et discret pendant que, vêtu jusqu'au cou, il contemple une nudité sans la toucher, la possédant à distance (96). Hilbert est décrit lui aussi comme 'vêtu jusqu'au cou', ayant même gardé ses gants ${ }^{38}$; il contemple pareillement une nudité sans la toucher, la possédant à distance (Sartre écrira même que Baudelaire mettait des gants pour faire l'amour, 97). Reprenant les préférences assignées à 
Baudelaire, Hilbert fait asseoir la prostituée sur le lit puis lui fait écarter les jambes. Il la regarde alors entre les cuisses avant de renifler puis de rire (87). 'Respirer la chair de la femme', voilà une caractéristique de l'érotisme sartrien ... que l'on retrouve dans sa correspondance. ${ }^{39} \mathrm{Le}$ scénario érotique continue : Hilbert donne ensuite une canne à la prostituée pour lui faire faire 'l'exercice' (87). La contemplation de cet exercice le fera jouir. Nous n'en saurons pas plus quant à cet exercice, mais une lecture intertextuelle nous renvoie à une autre nouvelle intitulée 'Dépaysement', écrite pendant l'été $1936^{40}$, où les gestes accomplis par deux prostituées avec 'un petit bâton blanc qui ressemblait à une bougie' seront décrits en détails. ${ }^{41}$

En choisissant cette forme particulière d'érotisme, Sade (ainsi que Baudelaire et Sartre) ont choisi 'l'imaginaire' (45). Sade écrit que 'la jouissance des sens est toujours réglée sur l'imagination' (45), un domaine interdit à ceux que Sartre appelle les paresseux d'esprit. Évoquant Sade, Beauvoir souligne que la représentation de la scène érotique a plus d'intérêt à ses yeux que son expérience vécue (43). Est-il besoin de le rappeler? Le Baudelaire de Sartre est voyeur et fétichiste. Selon l'auteur, ces vices 'réalisent la possession de loin, symboliquement, pour ainsi dire' (96). François Noudelmann rappelle que constituer l'autre ou soi-même en analogon aboutit à une fixation. Il explicite que l'imagination s'apparente alors au fétichisme, à l'incarnation déréalisante de certains objets, par lesquels le sujet trouve un mode de satisfaction qu'il ne cesse de répéter. Et ce mode conduit à une forme d'échec: 'L'imaginaire s'est transformé en un univers clos sur lui-même, une spirale qui entraîne le sujet dans une réflexion irréelle' ${ }^{2}{ }^{2}$

\section{Sartre et les femmes}

Comment les 'conquêtes' de Sartre réagissaient-elles à son érotisme? 'T. me voit en ce moment comme un bouc obscène ${ }^{43}$ apprend-on de la bouche même de Sartre en février 1940 dans ses 
Carnets de la drôle de guerre, des carnets destinés à Beauvoir qui les lisait au fur et à mesure. Que sait-on sur le bouc? Un bouc entier (non castré) est agressif au moment du rut. Sartre juge son comportement comme un défaut injustifiable, concluant qu'il se fait un peu horreur même s'il sait que ce reproche n'est pas bien juste, avant d'affirmer son désir de changer. ${ }^{44}$ Quant à ' $T$ ', c'est pourtant la seule qu'il ne gêne pas lors de leurs ébats amoureux, ce qu'il trouve comique. Dans un mea culpa épistolaire, il se décrit à Beauvoir comme un enfant vicieux dans ses rapports physiques, précisant qu'il a gêné les femmes de ce point de vue et même le Castor, surtout les premiers temps : 'vous m'avez un peu bien trouvé obscène. Non pas un bouc, certes. Cela je suis sûr de ne pas l'être. Mais obscène simplement'. ${ }^{45}$ Comparons ces propos avec une autre source, les Cahiers de jeunesse de Beauvoir. Elle raconte comment, en octobre 1929, Sartre lui dit que s'ils doivent renouveler leur bail [qui avait duré un an], il lui montrera 'des choses laides et viles en lui' ${ }^{46}$. Quelques jours plus tard, il semble que ce soit chose faite puisqu'elle lui déclare le trouver grand 'avec tout ce qu'il peut avoir de plus vil, de plus bas [...] de pensées basses' ${ }^{47}$. Elle précise un peu les choses en écrivant dans ses cahiers que 'même si c'était une servitude réelle de sa chair' qui lui était proposée par Sartre, elle accepterait ${ }^{48}$, cette dernière phrase faisant écho aux analyses du sadisme dans le traité philosophique. Si Sartre refuse la comparaison avec le bouc, il accepte malgré tout le qualificatif d'obscène, concédant qu'il y a quelque chose de très abîmé en lui. Il avoue qu'avec Martine Bourdin (Colette Gibert), qu'il ne respectait pas comme il pouvait respecter le Castor, qu'il ne ménageait pas comme il pouvait ménager T., il s'est conduit d'une manière abjecte ('mes rapports sexuels avec elle ont été ignobles' ${ }^{49}$ ), dans une atmosphère de 'canaillerie sadique', se traitant de 'Don Juan de village' et de 'sadique universitaire'; il avoue se sentir 'sali' par cette histoire. ${ }^{50}$ 
Et que fait remarquer Sartre au Castor dans cette même lettre (nous sommes en 1940)? Comme elle a pu le constater lors de sa dernière permission, il a changé pendant leurs rapports physiques: 'Peut-être la force des rapports physiques y perd-elle un peu mais je trouve qu'ils y gagnent en propreté'. ${ }^{51}$ Sa missive se termine par une affirmation qu'il apprend la pudeur à trente-quatre ans. Il n'y a qu'avec Beauvoir qu'il se sent propre, notant toutefois qu'il n'a pas reçu ce jour-là de lettre d'elle et que cela le laisse 'tout seul dans la merde'.${ }^{52}$ Il n'y a pas besoin d'être Sigmund Freud pour voir que toutes ces allusions se réfèrent au stade anal: sans Beauvoir, Sartre est littéralement dans la merde. Régis Debray rappelle que le premier sens du mot 'obscène' se réfère aux situations où s'exhibe ce que l'on doit cacher ou éviter et que le pluriel neutre, obscena, désigne les excréments. ${ }^{53}$ Beauvoir affirme que la sexualité sadienne est essentiellement anale $(37)^{54}$, analysant longuement la coprophilie de Sade au niveau de l'incarnation en expliquant que 'l'homme qui a commerce avec la saleté $[\ldots]$ se réalise comme chair' (38). Elle relie donc la coprophilie au problème philosophique de base: l'impossibilité pour Sade de réaliser la présence d'Autrui et de s'unir à lui/elle sans inventer des manières singulières de saisir l'autre.

C'est toujours vers les Lettres an Castor qu'il faut se tourner pour en savoir plus sur la 'canaillerie sadique' entre Sartre et Bourdin. Sartre décrit la jeune fille brune comme étant 'provençale comme le diable, pleine d'odeurs et curieusement velue, avec une petite fourrure noire au creux des reins'. Il parle de sensualité un peu violente qui au début l'a surpris voire un peu dégoûté. Maintenant qu'il s'y est habitué, 'c'est au contraire assez fort'. La description physique qu'il fait d'elle montre bien ses goûts esthétiques: 
Elle a des fesses en goutte d'eau, solides mais lourdes, plus étalées en bas qu'en haut [...] de très belles jambes, un ventre musclé et absolument plat, pas une ombre de poitrine et, dans l'ensemble, un corps souple et charmant. ${ }^{55}$

Sartre décrit un corps androgyne. L'important, ce sont les fesses et le fait qu'elle ait un corps souple, pour pouvoir le 'manier'.

Voici comment Sartre raconte l'histoire quelques jours plus tard. Tout d'abord, il lui assène de but en blanc, assis dans un café: "Alors [...] tu veux coucher avec moi. ${ }^{56}$ La nuit suivante, vers minuit, elle lui demande de la pénétrer avec son sexe. Alors qu'il s'exécute, elle geint. Il lui fait remarquer que si elle se caressait elle-même de la même manière qu'il la pénètre, elle mettrait son orgueil à ne pas crier mais comme elle a une peur vague, elle n'a pas honte de gémir. ${ }^{57}$ Quelques instants plus tard, elle lui dit d'arrêter. Il n'est question de jouissance ni pour l'un ni pour l'autre. Dans une lettre de rupture envoyée à Bourdin quelques mois plus tard, Sartre écrira l'avoir trouvée physiquement plaisante quoique vulgaire, (se) donnant l'explication suivante: 'j' ai un certain sadisme que ta vulgarité même attirait'. ${ }^{58}$

Au début Sartre ne veut pas la déflorer, puis il écrit à Beauvoir avoir pris sa virginité, décrivant 'un travail profondément difficile et désagréable'. ${ }^{59}$ Il ne faut pas oublier que Sartre couche 'tout' sur papier pour Beauvoir qui lui avait par ailleurs interdit de coucher avec Bourdin. ${ }^{60}$ Pour Doubrovsky, la jouissance de Sartre n'existe que 'dite-à-l'Autre', 'racontée au Castor', ${ }^{61}$ citant Buisine: 'On ne se met au lit que pour pouvoir ensuite se mettre dans la lettre'. ${ }^{62}$ Au-delà du contrat de transparence que Sartre et Beauvoir s'étaient donné comme ligne de conduite, au-delà de leur comportement aristocratique qui les fait ressembler aux libertins du XVIIIème siècle, que veut dire raconter $=($ re $)$ jouir? N'est-ce pas une posture littéraire, 
esthétisante: vivre comme au XVIIIème siècle $?^{63}$ Beauvoir souligne que Sade raconte ses fantaisies avant qu'elles ne soient pratiquées (43). Sartre, quant à lui, raconte ses ébats à Beauvoir après qu'ils soient pratiqués. Le résultat est le même: 'Par ce dédoublement l'acte devient un spectacle que l'on considère à distance dans l'instant où on l'exécute' (43) et liberté et conscience ne se perdent pas dans l'égarement de la chair (43).

Si Colette Gilbert (Martine Bourdin) n'a pas laissé sa version des faits, nous avons en revanche le témoignage de Lamblin. Ne laissant aucune place à la spontanéité, ils décident du jour et de l'heure et, alors qu'ils se dirigent vers la chambre d'hôtel, Sartre confie à Lamblin que la femme de chambre va être étonnée car la veille il a déjà pris la virginité d'une jeune fille. Arrivés dans la chambre, il se déshabille presque complètement avant de se laver les pieds dans le lavabo. Elle lui demande de tirer les rideaux pour atténuer la lumière. Il refuse sèchement, lui disant que ce qu'ils vont faire doit se faire en pleine lumière; elle se réfugie alors derrière les rideaux pour se déshabiller. Lamblin ne comprend pas l'attitude de Sartre: 'J'étais troublée et ne comprenais pas qu'il ait renoncé à son habituelle gentillesse; c'était comme s'il voulait brutaliser quelque chose en moi (mais aussi en lui-même), poussé par une impulsion destructrice'. ${ }^{64}$ Elle se rend très vite compte que son partenaire ne peut ni se laisser aller physiquement, ni s'abandonner à une émotion sensuelle. ${ }^{65}$ Cette attitude est à mettre en parallèle avec celle de Baudelaire. Sartre affirme que son désir est rigoureusement stérile et qu'il reste maitre de lui: 'Il s'agit d'une intention de désir, d'un fantôme de désir, plus que d'une réalité' (158). Beauvoir écrit que la jouissance chez Sade ne comporte 'ni échange, ni don, ni réciprocité, ni gratuite magnificence' (38). Nous trouvons de nouveau des similarités entre les trois écrivains. Lamblin raconte qu'elle était crispée et qu'aucune chaleur amoureuse, aucun geste spontané de son compagnon ne venaient adoucir la situation. Elle avait l'impression que Sartre suivait une sorte de programme 
déjà écrit, comparant ses actions à un acte chirurgical. ${ }^{66}$ Evoquant la cruauté de Sade, Beauvoir fait remarquer qu'elle ne couronne pas impulsivement un orgasme car elle tend de manière préméditée à le provoquer (41). C'est l'échec ce jour-là et Sartre s'empresse alors de lui faire un cours d'anatomie! À défaut de bouc obscène, Lamblin conclut sur 'le mélange de brutalité, de muflerie, de froideur physique, de pédantisme et de cuistrerie de Sartre' ${ }^{67}$

Toujours dans le même registre, dans une lettre, Sartre couche sur papier pour Beauvoir le dépucelage de Tania (T.) qu'il appelle une initiation, parlant de ce qu'il y a de 'ridicule pour un type dans une dévirginisation' puis parle des 'élucubrations' de Tania lorsque 'c'est fait' qui visent à 'être putain'. Il précise ces délires comme étant 'les plus hardies d'entôlage, de domination habile et sournoise, d'aventures étranges, etc. ${ }^{68}$ On se souviendra que T. était censée être la seule qui ne soit pas gênée par la conduite sexuelle de Sartre. Sartre, que l'on sache, raconte dans sa seule correspondance pour l'année 1939 avoir pris la virginité de quatre jeunes filles. Beauvoir explique que chez les héros de Sade, on s'amuse volontiers à dépuceler les fillettes (35). Pour Sade le rapt de virginité apparaît comme une cérémonie d'initiation dans la mesure où en violentant un individu on l'accule à assumer sa séparation et par là il trouve une vérité qui le réconcilie avec son antagoniste: 'Bourreau et victime se reconnaissent comme des semblables' (78).

Sans aller jusqu'au fouettage de Sade, Sartre relate une anecdote à Beauvoir dans sa correspondance sur celle qu'il appelle 'la petite Zazoulich': Olga Kosakiewicz. ${ }^{69}$ Il se présente comme un soldat au combat, révélant à Beauvoir comment, quand il la voit, il 'gagne chaque fois du terrain' ${ }^{70}$ Une journée de passion troublée où, dit-il, 'pour badiner', il lui a donné un léger coup sur les fesses, se termine ainsi: 'Elle a fait des manières, $\mathrm{j}$ 'ai grossi l'incident en drame, elle a sangloté et j'ai été ignoble'. ${ }^{71}$ Il tire le bilan suivant: 'Je suis tripoteur en titre, on parle même 
des baisers donnés entre les coups. Elle ne cache point le vif plaisir qu'elle y prend' ${ }^{72}$ Sartre écrit que pour Baudelaire 'faire souffrir c'est posséder et créer tout autant que détruire' (31). Dans L'Être et le Néant, le philosophe évoque l'incarnation de la douleur en tant que moyen de traiter autrui comme un instrument tout en étant aussi un moyen pour que le corps du Pour-soi, infligeant la douleur, devienne un instrument à donner de la douleur: 'Ainsi le Pour-soi dès l'origine peut se donner l'illusion de s'emparer instrumentalement de la liberté de l'Autre, c'està-dire couler cette liberté dans de la chair, sans cesser d'être celui qui provoque, qui empoigne, qui saisit, etc.' (450). Beauvoir démontre l'importance pour le sadique de recouvrer dans l'autre sa propre condition: il faut qu'il soit habité par une liberté et une conscience (34). Parlant de victimes et bourreaux, la philosophe souligne que les plaintes de la victime sont nécessaires au bonheur du bourreau car 'dans sa révolte, l'objet torturé s'affirme comme mon semblable et j'atteins par sa médiation cette synthèse de l'esprit et de la chair qui s'était d'abord refusée à moi' (34). Or, d'après Sartre, Olga prend un vif plaisir à ses coups. Dans ce cas-là, toujours suivant l'auteure de Faut-il brûler Sade?, si la victime tourne la souffrance en plaisir et la honte en orgueil, elle devient complice et le 'débauché' se trouve comblé. Sade est ensuite cité directement: 'Il n'est point pour un esprit libertin de plaisir plus vif que de faire des prosélytes' (78).

L'auteur du Mur (qui contient Erostrate) dédicacera son recueil de nouvelles à Olga. Doubrovsky analyse l'épisode où Sartre raconte à Beauvoir qu'il a couché avec son amie Gégé en s'arrêtant sur un fait: 'Elle m'a dit « J'ai eu presque du plaisir ». Alors je lui ai fait cadeau d'Erostrate pour la remercier' ${ }^{73}$ Encore faudrait-il s'arrêter sur la cause des remerciements: estce parce qu'elle a eu presque du plaisir ou est-ce pour s'excuser qu'elle n'ait pas eu de plaisir? Doubrovsky interprète ce geste de Sartre tendant Erostrate à Gégé (que nous pourrions étendre à 
Olga) comme une manière de dire 'Erostrate, c'est moi' (donc 'Paul Hilbert, c'est moi'): 'On retrouve, sous l'hyperbole délirante, le refus de pénétration sexuelle du corps féminin (travesti chez Sartre en dénigrement de l'acte), ainsi que le substitut de la possession physique sous forme de maîtrise sadique' ${ }^{74}$ Nous avons d'ores et déjà brossé le portrait du protocole à l'œuvre dans les récits des scènes érotiques que Sartre donne de ses conquêtes ou tel qu'il est reflété dans ses écrits, enrichi par Baudelaire ('Voir, palper, respirer la chair de la femme') ainsi qu'avec Beauvoir dans ses Cahiers de jeunesse qui imagine Sartre demandant 'une servitude réelle de sa chair'.

\section{Sadisme et liberté}

Le sadisme est-il un mode satisfaisant de vivre sa sexualité? Pour l'auteur de L'Être et le Néant, il renferme son propre échec:

Lorsque l'incarnation est achevée, lorsque j'ai bien devant moi un corps pantelant, je ne sais plus comment utiliser cette chair [...] elle est là et elle est là 'pour rien’ [...] Je ne puis que demeurer interdit devant elle [...] ou alors m'incarner à mon tour, me laisser prendre par le trouble [...] Ainsi le sadisme, au moment même où son but va être atteint, cède la place au désir. (455)

Selon Beauvoir, le tourmenteur réclame que la victime reconnaisse dans la liberté du tyran son destin, 'alors elle est unie à lui par le plus étroit des liens, ils forment véritablement un couple' (77). Toutefois, elle constate que la tentation sadique ne peut qu'aboutir à l'échec car si l'on a échoué à saisir dans une expérience vécue l'unité ambiguë de l'existence (faire coïncider 
l'intimité de la conscience avec l'opacité de la chair), on ne parviendra jamais à la reconstruire intellectuellement (44). Selon la philosophe, une fois dissociés, ces deux moments de la réalité humaine s'opposent et dès qu'on poursuit l'un, l'autre se dérobe. Elle utilise un oxymore sans équivoque: 'Où donc rencontrer de libres esclaves?' (44). En parallèle, Sartre conclut à propos de Baudelaire: 'Il veut être cette nature contradictoire: une liberté-chose' (84). Beauvoir souligne aussi qu'en souillant et blessant, le bourreau se souille et se blesse (39). Vient à l'esprit la phrase de Lamblin: 'c'était comme s'il voulait brutaliser quelque chose en moi (mais aussi en luimême)'. Pour Beauvoir, le bourreau finit par participer à cette passivité qu'il dévoile dans la mesure où il cherche à se saisir comme la cause des tourments qu'il inflige alors qu'en fait il se saisit en tant qu'instrument, donc en tant qu'objet. Ce raisonnement la pousse à qualifier ces conduites non seulement de sadisme mais également de sadomasochisme. Invoquant le motvalise 'castorisation' inventé par Buisine, Doubrovsky soutient que le sadisme sartrien trouve son envers masochiste lorsque Sartre présente Beauvoir comme son juge dans leur échange épistolaire et lui demande de ne surtout pas l'absoudre alors qu'il confesse ses tentations charnelles. ${ }^{75}$ Elle devient alors à ses yeux 'plus moi' que Jean-Paul: 'Elle est Surmoi [...] elle administre la Loi, Castor-Mentor et Juge suprême' ${ }^{76}$ Mais cette tactique ne serait-elle pas un exemple de qui perd gagne? Sartre écrit à propos de Baudelaire qu'il se constitue en objet par la sévérité sociale avec laquelle il se traite et devient du même coup son propre juge. L'échec de l'incarnation de la liberté d'Autrui dans l'acte sexuel est ici dépassé: 'la liberté s'échappe de la chose jugée pour venir imprégner l'accusateur' (106).

\section{L'unité ambiguë?}


Revenons au problème philosophique de la relation d'objet par le biais de la morale et notamment des Cahiers pour une morale de Sartre. Nous avons vu que Beauvoir s'intéresse à Sade car c'est quelqu'un qui 'de sa sexualité [a] fait une éthique, cette éthique il l'a manifestée dans une œuvre littéraire' (15). Elle n'hésite pas à écrire que Sade est un 'grand moraliste' (55). Dans un chapitre sur Sartre et Levinas, Howells montre combien, suite à la conversion annoncée dès L'Être et le Néant, Sartre est beaucoup plus optimiste dans sa description des relations humaines dans ses Cahiers pour une morale, publiés après sa mort, même s'ils ont été composés en 1947 et $1948 .{ }^{77}$ Au niveau biographique, Sartre a toujours dit que la Seconde Guerre mondiale avait divisé sa vie en deux et qu'il était passé de l'individualisme et de l'individu au social et au socialisme. ${ }^{78}$ Cette évolution semble se retrouver dans la relation avec Autrui et il est à noter que tous les exemples de relations sexuelles que nous avons donnés se situent à la fin des années trente ou pendant la guerre. Sur un plan théorique, au niveau de la conscience, ce nouveau Sartre d'après-guerre accepte désormais que l'on se crée soi-même en se donnant à l'autre: 'Ainsi doisje me perdre pour me trouver'. ${ }^{79}$ Ceci nous renvoie à Bersani pour qui, mis à part Freud et Lacan, toutes les autres théories sur l'amour partagent une même assomption: 'In love, the human subject is exceptionally open to otherness' ${ }^{80}$ Howells cite un autre passage important des Cahiers où Sartre affirme que 'l'Ego est pour se perdre: C'est le Don', ${ }^{81}$ l'Ego se perd dans la dimension absolue de la liberté ${ }^{82}$ avant de se focaliser également sur un passage des Cahiers où Sartre traite explicitement du sadisme et du masochisme ${ }^{83}$ Suite à la conversion (en un mot entendons le rejet de l'aliénation $)^{84}$, ces deux concepts n'ont plus de sens dans la mesure où l'on a accepté d'être à la fois liberté et objet pour autrui et de dépasser cet être-objet. Sartre revient sur ce qu'il nomme 'la dialectique sadico-masochiste d'asservissement' telle qu'il l'a décrite dans $L$ 'Etre et le Néant pour dire qu'elle entrave l'amour qui ne peut naître que dans une 
reconnaissance mutuelle. Mais il pense toujours, ajoute-t-il, que cette dialectique doit être présente en tant que tension car, sans elle, l'amour - qu'il qualifie de 'sexuel' - disparaît. On pourrait croire avec cette dernière remarque que sa position n'a pas vraiment changé depuis L'Être et le Néant, or il précise sa pensée en affirmant que cette tension maintient les deux faces de l'ambiguïté et les retient dans l'unité d'un même projet et que, dès qu'on lâche l'ambiguïté, la dualité reprend, concluant: 'Il faut inventer'. ${ }^{85}$ Avec le concept de narcissisme impersonnel, Bersani fondait le nouveau pouvoir de l'amour en n'utilisant plus l'opposition entre le même et l'autre exprimée comme différence en tant que structure catégorisant les êtres humains. ${ }^{86}$ Sartre fonde toujours l'unité à partir d'une tension basée sur l'ambiguïté de la dyade sujet-objet et cette tension est la garantie de l'unité qui s'effondre en dualité dès qu'elle disparaît. Bersani prend le problème à l'envers en ne qualifiant plus de différence la relation avec Autrui.

En nous appuyant notamment sur Baudelaire et sur Faut-il brûler Sade?, notre article a permis de mesurer l'importance de l'imaginaire chez les trois auteurs étudiés, de creuser et de préciser les notions de désir, d'érotisme ainsi que de sadisme chez Sartre en esquissant le protocole à l'œuvre dans les scènes érotiques, guidés par Doubrovsky et par Howells. Ce accomplissant, nous a permis de mieux apprécier l'importance métaphysique de certains passages de sa fiction comme la nouvelle Erostrate voire certains comportements de Sartre comme celui raconté par Bianca Lamblin ou ceux qu'il relate lui-même par rapport à Olga, Tania ou Colette Gibert en nuançant le côté sensationnaliste et en posant des bases philosophiques. Nous pouvons désormais établir que le Sartre des Cahiers, le Sartre d'après-guerre, s'est rapproché de la position de Beauvoir dans Faut-il brûler Sade? Cette dernière affirme que, par le trouble, l'existence est saisie en soi et chez l'autre comme subjectivité et passivité à la fois. Il en résulte qu' 'à travers cette unité ambiguë les deux partenaires se confondent; chacun est délivré 
de sa présence à soi et atteint une communication immédiate avec l'autre' [nous soulignons] (33), deux sujets arrivant à coexister dans la jouissance. Dans les débats sur l'influence mutuelle entre Sartre et Beauvoir, cette pièce est à verser au dossier en faveur de Beauvoir.

Reste à savoir si Sartre mit ces notions théoriques en pratique dans sa vie érotique à partir des années 1950 ou s'il continua à perpétuer ce qu'Howells appelle les tourniquets sadomasochistes de 1'amour et du désir. ${ }^{87}$

Jean-Pierre Boulé est professeur d'Études françaises contemporaines à l'université de Nottingham Trent. Il est l'auteur de Sartre médiatique (1992) et Sartre, Self-Formation and Masculinities (2005) et co-éditeur de Jean-Paul Sartre: Mind and Body, Word and Deed (2011), Existentialism and Contemporary Cinema: A Sartrean Perspective (2011) et Existentialism and Contemporary Cinema: A Beauvoirian Perspective (2012). Il a été l'éditeur de Sartre Studies International de 2008 à 2014.

\footnotetext{
Abstract: This article proposes a focus on eroticism and the sexual act in the work and biography of Sartre, particularly on the question of sadism, exploring the thesis that Sartre's biography of Charles Baudelaire and Simone de Beauvoir's essay on the Marquis de Sade indirectly represent and help us to explore Sartre's own eroticism, desire and sadism. It is characterized by references to fictional and nonfictional works alike, and to Sartre's correspondence and journals. The analysis is also supported through a wide range of secondary critical sources, in particular articles by Christina Howells and Serge Doubrovsky. The focus on the physical materiality of sex and eroticism is accompanied by a strong thread of attention to the existential and psychological structures of sadism and masochism.
} 
Keywords: Charles Baudelaire, eroticism, Jean-Paul Sartre, Marquis de Sade, object relations theory, sadism, Simone de Beauvoir

Jean-Pierre Boulé is professor of contemporary French studies at the Nottingham Trent University. He is the author of Sartre médiatique (1992) and Sartre, Self-Formation and Masculinities (2005) and co-editor of Jean-Paul Sartre: Mind and Body, Word and Deed (2011), Existentialism and Contemporary Cinema: A Sartrean Perspective (2011) and Existentialism and Contemporary Cinema: A Beauvoirian Perspective (2012). He served as executive editor of Sartre Studies International from 2008 to 2014.

\section{Notes}

${ }^{1}$ Jean-Paul Sartre, Situations, X, Politique et Autobiographie (Paris: Gallimard, 1976), 147.

${ }^{2}$ Christina Howells, 'Sartre: Desiring the Impossible', in Philosophy and Desire, ed. Hugh J.

Silverman (London: Routledge, 2000): 85-95 (91).

${ }^{3}$ Serge Doubrovsky, 'Sartre: Retouches à un autoportrait (une autobiographie visqueuse)', in Lectures de Sartre, ed. Claude Burgelin (Lyon: Presses universitaires de Lyon, 1986), 99-134.

${ }^{4}$ Sans nommer précisément le domaine de la sexualité, Buisine parle des projections de Sartre dans ses biographies existentielles de Baudelaire, Genet et Flaubert. Alain Buisine, Laideurs de Sartre (Lille: Presses universitaires de Lille, 1986), 42n4. Voir également Josette Pacaly, Sartre au miroir: Une lecture psychanalytique de ses écrits biographiques (Paris: Klincksieck, 1980). ${ }^{5}$ Jean-Paul Sartre, Baudelaire (Paris: Gallimard, 1963); Simone de Beauvoir, Faut-il brûler Sade? (Paris: Gallimard, 1955). (Afin d'alléger les notes, nous inscrirons la pagination de ces deux ouvrages dans le texte entre parenthèses.) Professor Debra Bergoffen a ouvert cette piste de 
recherches en me suggérant lors d'une conversation que ce texte sur Sade pourrait refléter une approche de la sexualité chez Sartre. Dans son livre, Debra B. Bergoffen, The Philosophy of Simone de Beauvoir: Gendered Phenomenologies, Erotic Generosities (Albany: State University of New York Press, 1997), Bergoffen arguë que Beauvoir ne peut pas être lue sans tenir compte de son texte sur le marquis de Sade (12) - voir son chapitre: 'Perversions: "Must we Burn Sade?", 113-138.

${ }^{6}$ Pierre Klossowski, Sade mon prochain (Paris: Seuil, 1947).

${ }^{7}$ Maurice Blanchot, Lautréamont et Sade (Paris: Éditions de Minuit, 1949). Une partie de ce livre 'La raison de Sade', un compte-rendu du livre de Klossowski, avait été publiée dans Les Temps modernes en 1947 sous le titre 'À la rencontre de Sade', la revue dirigée par Jean-Paul Sartre où Beauvoir faisait partie du comité de rédaction.

${ }^{8}$ Voir par exemple 'Sade, l’homme souverain' dans Georges Bataille, L'Érotisme (Paris: Éditions de Minuit, 1957).

${ }^{9}$ Ursula Tidd, 'Devenir Mère: Trajectories of the Maternal bond in Recent Films Starring Isabelle Huppert', in Existentialism and Contemporary Cinema: A Beauvoirian Perspective, ed. Jean-Pierre Boulé and Ursula Tidd (Oxford: Berghahn Books, 2012), 33-51 (47).

${ }^{10}$ Judith Butler, 'Beauvoir on Sade: Making Sexuality into an Ethic', in The Cambridge Companion to Simone de Beauvoir, ed. Claudia Card (Cambridge: Cambridge University Press, 2003), 168-188 (186).

${ }^{11}$ Bergoffen, The Philosophy of Simone de Beauvoir, 116.

${ }^{12}$ Ibid., 137. 
${ }^{13}$ Jean-Paul Sartre, L'Être et le Néant (Paris: Gallimard, 1943). Édition renouvelée en 1970, 413. Afin d'alléger les notes, nous inscrirons la pagination dans le texte entre parenthèses. Si le contexte n'est pas clair, nous utiliserons l'abréviation $E \& N$.

${ }^{14}$ Leo Bersani and Adam Phillips, Intimacies (ReadHowYouWant edition: Accessible Publishing Systems PTY, 2010), 73-74.

${ }^{15}$ Sigmund Freud, Trois essais sur la théorie sexuelle (Paris: Gallimard, 'Folio', 1989).

${ }^{16}$ Bersani and Phillips, Intimacies, 74.

${ }^{17}$ Jacques Lacan, Les Quatre concepts fondamentaux de la psychanalyse (Paris: Points, 2014).

${ }^{18}$ Bersani and Phillips, Intimacies, 88-89.

19 'C'est cet autisme qui lui interdit de jamais s'oublier et de jamais réaliser la présence d'autrui' (33). Pour Butler l'autisme est le corrélat psychique du solipsisme et c'est ce solipsisme qui empêche Sade de reconnaître l'Autre. 'Beauvoir on Sade', 185.

${ }^{20}$ Gayle S. Rubin, Deviations (Durham, NC: Duke University Press, 2011).

${ }^{21}$ Simone de Beauvoir, La Cérémonie des adieux suivi de Entretiens avec Jean-Paul Sartre (Paris: Gallimard, 1981), 163-559 (400). Afin d'alléger les notes, nous inscrirons la pagination dans le texte entre parenthèses.

${ }^{22}$ Jean-Paul Sartre, L’Âge de raison (Paris: Gallimard, 1945), édition Folio, réimprimé 1980, 20.

${ }^{23}$ Howells, 'Sartre: Desiring the Impossible', 89.

24 'Pour une psychologie de l'homme féodal', Les Temps Modernes, nos 645-646 (2007): 76$123(101)$.

${ }^{25}$ Simone de Beauvoir, Cahiers de jeunesse (Paris: Gallimard, 2008), 798.

${ }^{26}$ Michel Contat, 'Sartre and His Other Women', Journal of Romance Studies 6, nos 1-2 (2006): $115-124(118)$. 
${ }^{27}$ Bianca Lamblin, Mémoires d'une jeune fille dérangée (Paris: Balland, 1993), 56.

${ }^{28}$ Simone de Beauvoir, Lettres au Castor et à quelques autres, ** (Paris: Gallimard, 1983), 93.

${ }^{29}$ Doubrovsky, 'Sartre: Retouches à un autoportrait', 116.

${ }^{30}$ Leo Bersani, 'Is the Rectum a Grave?', in AIDS, Cultural Analysis, Cultural Activism, ed. Douglas Crimp (London: MIT Press, 1988), 197-222 (218).

${ }^{31}$ Pour un article critique, voir Sharon Ghamari-Tabrizi, 'Feminine Substance in Being and Nothingness', in Jean-Paul Sartre, ed. Harold Boom (Philadelphia: Chelsea House Publishers, 2001), 189-198. 'Sartre's fantasy of engulfment by the world translates into anxiety of overidentifying with the feminine', 192.

${ }^{32}$ Ibid., 118.

${ }^{33}$ Doubrovsky, 'Sartre: Retouches à un autoportrait', 118

${ }^{34}$ Jean-Paul Sartre, Le Mur (Paris: Gallimard, 1939) réimpression en 1978, 85-88.

${ }^{35}$ Ibid., 86.

${ }^{36}$ Ibid., 88.

${ }^{37}$ Ibid., 83-84.

${ }^{38}$ Ibid., 86.

${ }^{39}$ Dans une lettre au Castor, Sartre décrit son repas comprenant un 'filet de barbue Bercy qui sentait la femme à s'y méprendre, au point que j'en ai bandé à demi'. Simone de Beauvoir, Lettres au Castor et à quelques autres, * (Paris: Gallimard, 1983), 104.

${ }^{40}$ Jean-Paul Sartre, Euvres romanesques (Paris: Gallimard, La Pléiade, 1981), 1537-1555.

${ }^{41}$ Analysant cette scène, Pacaly souligne le fait que les deux prostituées sont utilisées pour un culte phallique et que toute féminité leur est refusée. Pacaly, Sartre au miroir, 298.

${ }^{42}$ François Noudelmann, Sartre: L'incarnation imaginaire (Paris: L'Harmattan, 1996), 206. 
${ }^{43}$ Jean-Paul Sartre, Les Carnets de la drôle de guerre, Novembre 1939-Mars 1940 (Paris:

Gallimard, 1983), 295. Il s'agit de Tania, dans la vie: Wanda Kosakiewicz.

${ }^{44}$ Ibid., 261.

${ }^{45}$ Beauvoir, Lettres au Castor et à quelques autres, **, 93.

${ }^{46}$ Beauvoir, Cahiers de jeunesse, 797.

${ }^{47}$ Ibid., 804.

${ }^{48}$ Ibid., 794.

${ }^{49}$ Beauvoir, Lettres au Castor et à quelques autres, **, 93.

${ }^{50}$ Ibid., 94.

${ }^{51}$ Ibid., 93.

${ }^{52}$ Ibid., 95.

${ }^{53}$ Régis Debray, L'Obscénité démocratique (Paris: Café Voltaire, 2007), 31. Obscena désigne également les parties viriles.

${ }^{54}$ À ce sujet on lira aussi Roland Barthes, Sade, Fourier, Loyola (Paris: Éditions du Seuil, 1971).

${ }^{55}$ Beauvoir, Lettres au Castor et à quelques autres, *, 188.

${ }^{56}$ Ibid., 194.

${ }^{57}$ Ibid., 199.

${ }^{58}$ Beauvoir, Lettres au Castor et à quelques autres, **, 90.

${ }^{59}$ Beauvoir, Lettres au Castor et à quelques autres, *, 190.

${ }^{60}$ Ibid., 192.

${ }^{61}$ Doubrovsky, 'Sartre: Retouches à un autoportrait', 113. On sait pourtant que Beauvoir a censuré certaines de ces lettres avant de les préparer pour la publication.

${ }^{62}$ Buisine, 'Ici Sartre’, Revue des sciences humaines 66, no. 195 (1984): 183-203 (189). 
${ }^{63}$ Je dois cette idée à Georges Barrère - qui a aussi relu une première version de ce texte et a fait de nombreuses suggestions.

${ }^{64}$ Lamblin, Mémoires d'une jeune fille dérangée, 55.

65 Ibid., 56.

66 Ibid., 57.

${ }^{67}$ Ibid., 58.

${ }^{68}$ Beauvoir, Lettres au Castor et à quelques autres, *, 239.

${ }^{69}$ Ibid., 201.

${ }^{70}$ Ibid., 202.

${ }^{71}$ Ibid., 203.

72 Ibid., 204.

${ }^{73}$ Ibid., 136.

${ }^{74}$ Doubrovsky, 'Sartre: Retouches à un autoportrait', 122.

${ }^{75}$ Ibid., 131.

${ }^{76}$ Ibid., 130.

${ }^{77}$ Christina Howells, 'Sartre and Levinas', in The Provocation of Levinas: Rethinking the Other, ed. Robert Barnasconi and David Wood (London: Routledge, 1988), 91-99 (96).

${ }^{78}$ Sartre, Situations $X, 180$.

${ }^{79}$ Jean-Paul Sartre, Cahiers pour une morale (Paris: Gallimard, 1983), 136.

${ }^{80}$ Bersani and Phillips, Intimacies, 77.

${ }^{81}$ Sartre, Cahiers pour une morale, 434.

${ }^{82}$ Howells pense que Sartre n'a peut-être pas voulu publier ses Cahiers pour une morale de son vivant car on aurait pu l'accuser d'utopie. Howells, 'Sartre and Levinas', 97. 
${ }^{83}$ Sartre, Cahiers pour une morale, 26.

${ }^{84}$ Ibid., 486. Pour une définition du concept de conversion, voir François Noudelmann et Gilles

Philippe, Dictionnaire Sartre (Paris: Honoré Champion, 2004), 108-109.

${ }^{85}$ Sartre, Cahiers pour une morale, 430.

${ }^{86}$ Bersani and Phillips, Intimacies, 88-89.

${ }^{87}$ Howells, 'Sartre: Desiring the Impossible', 93. 